\title{
Handheld Capillary Blood Lactate Analyzer as an Accessible and Cost-Effective Prognostic Tool for the Assessment of Death and Heart Failure Occurrence during Long-Term Follow-Up
}

\author{
Grzegorz M. Kubiak, ${ }^{1,2}$ Wojciech Jacheć, ${ }^{1,3}$ \\ Celina Wojciechowska, ${ }^{1,3}$ Magdalena Traczewska, ${ }^{1,2}$ Agnieszka Kolaszko, ${ }^{1}$ \\ Leszek Kubiak, ${ }^{2}$ Joanna Jojko, ${ }^{1}$ and Ewa Nowalany-Kozielska ${ }^{1,3}$ \\ ${ }^{1}$ Clinical Department of Cardiovascular Diseases, Multidisciplinary Tertiary Hospital, Medical University of Silesia, \\ 10 Curie-Skłodowska Street, 41-800 Zabrze, Poland \\ ${ }^{2}$ Internal Medicine Department, Pyskowice Municipal Hospital, 2 Szpitalna Street, 44-120 Pyskowice, Poland \\ ${ }^{3} 2 n d$ Department of Cardiology in Zabrze, Medical University of Silesia, Katowice, Poland
}

Correspondence should be addressed to Grzegorz M. Kubiak; greg_kubiak@yahoo.com

Received 12 October 2016; Revised 26 November 2016; Accepted 6 December 2016

Academic Editor: Ying Huang

Copyright ( 2016 Grzegorz M. Kubiak et al. This is an open access article distributed under the Creative Commons Attribution License, which permits unrestricted use, distribution, and reproduction in any medium, provided the original work is properly cited.

\begin{abstract}
Impact of tissue lactate accumulation on prognosis after acute myocardial infarction (AMI) is biased. The study aimed to assess the prognostic role of lactate concentration (LC) in patients with AMI during one year of follow-up. 145 consecutive patients admitted due to AMI were enrolled. The data on the frequency of endpoint occurrence (defined as I, death; II, heart failure (HF); and III, recurrent myocardial infarction (re-MI)) were collected. The patients were divided into group A (LC below the cut-off value) and group B (LC above the cut-off value) for the endpoints according to receiver operating characteristic (ROC) analysis. The cumulative survival rate was $99 \%$ in group I-A and $85 \%$ in group I-B ( $p=0.0004, \log$-rank test). The HF-free survival rate was $95 \%$ in group II-A and $82 \%$ in group II-B ( $p=0.0095$, log-rank test). The re-MI-free survival rate did not differ between groups. A multivariate Cox analysis showed a statistically significant influence of LC on death [Hazard Ratio (HR): 1.41, 95\% Confidence Interval (CI) (1.13-1.76), and $p=0.002$ ] and HF [HR: 1.21, 95\% CI (1.05-1.4), and $p=0.007$ ] with no impact on re-MI occurrence. LC in capillary blood may be considered a useful prognostic marker of late-onset heart failure and death after AMI.
\end{abstract}

\section{Introduction}

Cardiovascular diseases are the most common cause of death in Poland, with an annual death rate of 452 per 100,000. Cardiovascular diseases thus represent a serious health problem for the country [1]. The morbidity rate of acute myocardial infarction (AMI), as shown by Yeh et al. [2], showed a tendency to decrease systematically. Irrespective of a broad spectrum of activities, a further decrease in the mortality rate primarily depends on innovation in pharmacotherapy and primary percutaneous coronary intervention (PCI) delay shortening. The abovementioned actions may positively impact the AMI in-hospital mortality rates being reported in
Europe as 7\% in the STEMI and 3-5\% in the NSTEMI group of patients [3-6]. Patient-dependent delay plays a crucial role in the potential improvement of AMI treatment results; there is thus a need for the implementation of an easy-touse and cost-effective diagnostic and prognostic tool. AMI is associated with an anaerobic switch phenomenon, which is primarily due to coronary artery occlusion, leading to the acute impairment of myocardium oxygen supply. A deterioration in left ventricle contractility leading to a reduction in cardiac output may result in peripheral tissue hypoxemia and inhibit glycolysis, which represents the primary source of adenosine triphosphate (ATP) supply, providing thirtyeight moles of ATP from one molecule of glucose. Pyruvate 
TABLE 1: Patient characteristics according to the type of myocardial infarction.

\begin{tabular}{|c|c|c|c|}
\hline & STEMI $(n=60)$ & NSTEMI $(n=85)$ & $p$ \\
\hline Age & 59.85 & 62.86 & ns \\
\hline Women & $21(35 \%)$ & $25(30 \%)$ & ns \\
\hline Hypertension & $50(83 \%)$ & $76(89 \%)$ & ns \\
\hline Diabetes & $20(33 \%)$ & $20(24 \%)$ & ns \\
\hline Smoking & $36(60 \%)$ & $37(44 \%)$ & ns \\
\hline Atrial fibrillation & $5(8 \%)$ & $6(7 \%)$ & ns \\
\hline Systolic BP (mmHg) & 136 & 140 & ns \\
\hline Diastolic BP (mmHg) & 81 & 83 & ns \\
\hline Shock & $7(12 \%)$ & $1(1 \%)$ & ns \\
\hline IABP & $2(3 \%)$ & $0(0 \%)$ & ns \\
\hline $\mathrm{eGFR}<60\left(\mathrm{ml} / \mathrm{min} / 1.73^{2}\right)$ & $5(8 \%)$ & $11(13 \%)$ & ns \\
\hline Previous CABG & $1(2 \%)$ & $3(4 \%)$ & ns \\
\hline Previous cardiac arrest & $3(5 \%)$ & $2(2 \%)$ & ns \\
\hline GPIIb/IIIa inhibitors & $29(48 \%)$ & $8(9 \%)$ & $p<0.001$ \\
\hline $\mathrm{EDV}(\mathrm{ml})$ & 97 & 90 & ns \\
\hline $\operatorname{LVEF}(\%)$ & 42 & 49 & $p<0.005$ \\
\hline Hospitalization time (days) & 8.8 & 8.0 & ns \\
\hline Heart rate $(1 / \mathrm{min})$ & 78 & 82 & ns \\
\hline BMI & 27.7 & 28.6 & ns \\
\hline Time from symptom onset to FMC (hours) & $7.77 \pm 7.19 ; 6.00(3.25,9.00)$ & $22.04 \pm 21.68 ; 12.00(8.00,24.00)$ & $p<0.001$ \\
\hline
\end{tabular}

Abbreviations: BMI: body mass index. BP: blood pressure. CABG: coronary artery bypass grafts. EDV: end-diastolic volume. eGFR: estimated glomerular filtration rate. GPIIb/IIIa: glycoprotein IIb/IIIa inhibitors. IABP: intra-aortic balloon pump. LVEF: left ventricular ejection fraction. NSTEMI: non-ST segment elevation myocardial infarction. STEMI: ST segment elevation myocardial infarction.

reduction into lactate restores only two moles of ATP in anaerobic conditions and is thus largely ineffective. Lactate was discovered by Scheele [7] in 1780 and was implemented into a clinical setting more than sixty years later by Johann Scherer [8] for various methods of assessment including a capillary blood handheld analyzer. The accumulation of lactate increases tissue acidosis with subsequent acid-base homeostasis disturbance, resulting in extreme conditions and symptoms of shock (tachycardia, tachypnea, cyanosis, pallor, third heart sound, and cold extremities) [9]. Whether the type of shock (hypovolemic, septic, or cardiogenic) differentially impacts the lactate concentration (LC) remains to be addressed with appropriate randomized multicenter studies.

\section{Aim of the Study}

Aim of the study was to assess LC in AMI patients; to evaluate the potential relationships between LC and other clinical and biochemical factors; to assess the possible prognostic impact of LC on death, heart failure (HF), and recurrent myocardial infarction (Re-MI) occurrence in one year of follow-up.

\section{Methodology and Patients}

We enrolled 145 consecutive patients into a prospective cohort study. They were admitted to our center (a tertiary university hospital) due to AMI between August and December 2012 and in them we performed LC measurement using the handheld The Edge ${ }^{\circledR}$ device (provided by the APEXBIO Company, Taiwan). The total cost of the equipment, including the electronic analyzer device and the measurement strips, was 410 USD, which equals 2.83 USD per patient. The study complies with the Declaration of Helsinki and was approved by the local ethics committee (approval number KNW/0022/KB1/99/12), and all patients gave informed consent prior to enrollment. The inclusion criteria included AMI according to the current European Society of Cardiology (ESC) definition [4] and age between 18 and 80 years. All enrolled patients were submitted for invasive coronary angiography. The exclusion criteria were a lack of informed consent (unconscious patients) or known malignant disease. Patients received typical treatment according to the current ESC guidelines, which were independent of the results of the LC measurement and were submitted for revascularization via percutaneous coronary intervention (PCI) coronary artery bypass grafts (CABG) or optimal medical therapy (OMT) either ad hoc or after a heart team decision process. The enrolled patients were subsequently analyzed according to the defined endpoints, which included death, HF, and re-MI occurrence in one year of follow-up performed in the majority of cases during clinical examination $(90 \%)$ or telephone survey (10\%). Nevertheless each endpoint which occurred during follow-up had to be confirmed by the data received from the national health care system digital database. The implemented system of blinded control during follow-up tended to improve the objectivity of the acquired data. The patients characteristics divided into STEMI and NSTEMI group were presented in the Table 1. 
TABLE 2: Biochemical parameters in STEMI versus NSTEMI patients.

\begin{tabular}{|c|c|c|c|}
\hline Parameter & STEMI $(n=60)$ & NSTEMI $(n=85)$ & $p$ \\
\hline Lactate concentration $(\mathrm{mmol} / \mathrm{l})$ & 4.0 & 4.1 & ns \\
\hline Glycemia (mmol/l) & 8.4 & 7.3 & $p<0.001$ \\
\hline Total cholesterol (mg/dl) & 220 & 215 & ns \\
\hline Triglycerides (mg/dl) & 126 & 124 & ns \\
\hline HDL (mg/dl) & 60 & 55 & ns \\
\hline $\mathrm{LDL}(\mathrm{mg} / \mathrm{dl})$ & 135 & 136 & ns \\
\hline $\mathrm{cTn}(\mathrm{ng} / \mathrm{l})$ & 847 & 357 & ns \\
\hline CK-MB (IU/l) & 64 & 49 & ns \\
\hline Creatinine (umol/l) & 78 & 81 & ns \\
\hline $\operatorname{RBC}\left(10^{6} / \mathrm{ul}\right)$ & 4.8 & 4.8 & ns \\
\hline WBC $\left(10^{3} / \mathrm{ul}\right)$ & 12 & 10 & $p<0.025$ \\
\hline $\operatorname{PLT}\left(10^{3} / \mathrm{ul}\right)$ & 259 & 239 & ns \\
\hline $\operatorname{HGB}(\mathrm{g} / \mathrm{dl})$ & 14 & 14 & ns \\
\hline
\end{tabular}

CK-MB: creatine kinase MB. cTn: cardiac troponin. HDL: high-density lipoproteins. HGB: hemoglobin. LDL: low-density lipoproteins. NSTEMI: non-ST elevation myocardial infarction. PLT: platelet count. RBC: red blood cell count. STEMI: ST elevation myocardial infarction.

\section{Statistical Analysis}

The distributions of the examined parameters were analyzed using the Shapiro-Wilk test. Values were presented as the means and standard deviation (SD) or as the median in the 25th and 75th percentiles. Nominal and categorical values were expressed in percentages or proportional rates. Linear variables with a normal distribution were compared using Student's $t$-test. Variables with an abnormal distribution were compared using the Kolmogorov-Smirnov and MannWhitney $U$ tests. Categorical variables of abnormal distribution were compared using a Chi-square test with Yates correction. A Kaplan-Meier analysis (log-rank test) was used to demonstrate the frequency of endpoint occurrence during the follow-up period for the patients, which were divided into two groups with low (A) and high (B) LC according to the calculated cut-off value (acquired from receiver operating characteristic (ROC) analysis). Cut-off values for each endpoint were calculated using ROC with subsequent sensitivity and specificity, area under curve (AUC), and Confidence Interval estimation. Cox proportional hazard regression was used to evaluate the risk of endpoint occurrence. The independent variables included in the multivariate model were variables that reached statistical significance (set at 0.1) in the univariate analysis of all parameters. Independent predictors of endpoint occurrence were presented as the Hazard Ratio (HR) with a Confidence Interval (CI). Differences between the values were considered statistically significant if $p<0.05$. Analyses were performed using Statistica 10 with the medical package (StatSoft Inc.).

\section{Results}

STEMI patients are characterized by the more frequent use of GPIIb/IIIa receptor blockers (48 versus 9\%, $p<0.001$ ), lower baseline left ventricular ejection fraction (LVEF) (42 versus $49 \%, p<0.005)$, and shorter time from the onset of symptoms to first medical contact (FMC) (8 versus 22

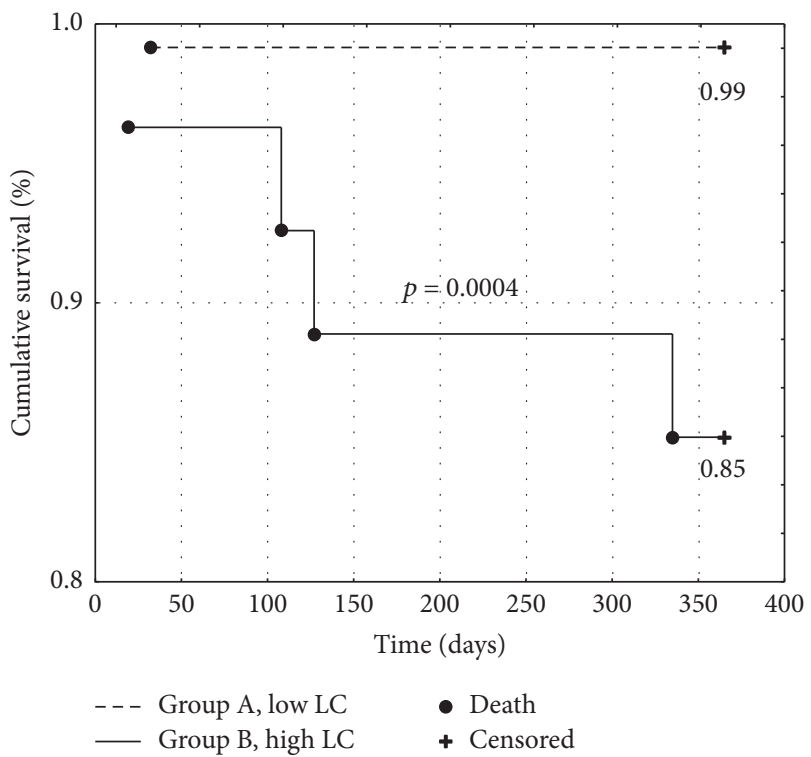

FIgURE 1: Kaplan-Meier survival curves for all-cause mortality of patients with low (group A) versus high (group B) lactate concentration. LC: lactate concentration.

hours, $p<0.001)$. Patient characteristics according to AMI type are presented in Table 1. STEMI patients had a higher serum blood concentration of glucose $(8.4$ versus $7.3 \mathrm{mmol} / \mathrm{l}$, $p<0.001$ ) and white blood cell count (12 versus $10 * 10^{3} / \mathrm{ul}$, $p<0.025)$. Data are presented in Table 2.

The highest AUC of 0.9 (with a specificity and sensitivity of $80 \%$ at the LC cut-off value of $5.35 \mathrm{mmol} / \mathrm{l}$ ) was observed for the death endpoint; this was marked as I. All data are presented in Table 3

Kaplan-Meier curves were used to assess the cumulative event-free survival for each endpoint. Patients with an LC below the cut-off value had a significantly higher cumulative survival rate $(0.99$ versus $0.85, p<0.001$, Figure 1$)$ and 
TABLE 3: ROC-derived cut-off values of LC for each endpoint.

\begin{tabular}{|c|c|c|c|c|}
\hline Endpoint during follow-up & $\mathrm{LC}(\mathrm{mmol} / \mathrm{l})$ & Sensitivity and specificity (\%) & $\mathrm{AUC} \pm \mathrm{SD}$ & $95 \% \mathrm{CI}$ \\
\hline Death (I) & 5.35 & 80 & $0.9 \pm 0.05$ & $(0.80-0.99)$ \\
\hline Heart failure (II) & 4.14 & 69 & $0.74 \pm 0.07$ & $(0.61-0.88)$ \\
\hline Re-MI (III) & 3.28 & 49 & $0.56 \pm 0.07$ & $(0.43-0.69)$ \\
\hline
\end{tabular}

AUC: area under the curve. CI: confidence interval. LC: lactate concentration. Re-MI: recurrent myocardial infarction. ROC: receiver operating characteristic. SD: standard deviation.

TABLE 4: Cox regression model for death occurrence in one year of follow-up.

\begin{tabular}{lcccccc}
\hline \multirow{2}{*}{ Endpoint I } & \multicolumn{3}{c}{ Univariate Cox regression analysis } & \multicolumn{2}{c}{ Multivariate Cox regression analysis } \\
& HR & $95 \%$ CI & $p$ & HR & - & - \\
HGB & 0.68 & $(0.47-0.97)$ & 0.030 & CI & - \\
LC & 1.29 & $(1.11-1.51)$ & 0.001 & 1.41 & $(1.13-1.76)$ & 0.002 \\
LDL & 0.98 & $(0.97-0.99)$ & 0.004 & 0.97 & $(0.95-0.99)$ \\
LM $>$ 50\% & 7.16 & $(1.15-44.47)$ & 0.031 & 11.06 & $(1.34-91.04)$ \\
\hline
\end{tabular}

HGB: hemoglobin. LC: lactate concentration. LDL: low-density lipoproteins. LM: left main coronary artery.

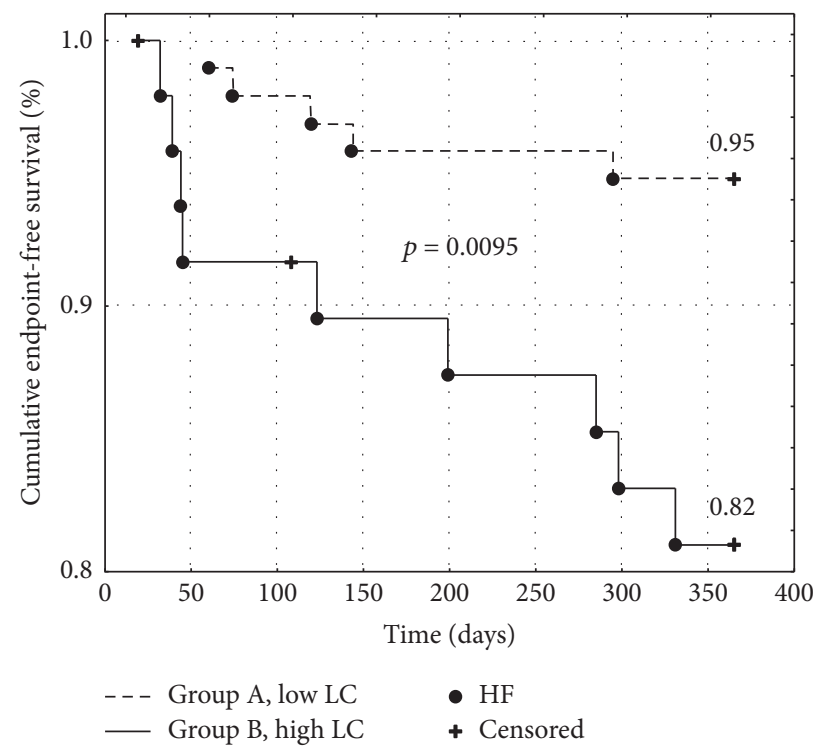

FIGURE 2: Kaplan-Meier event-free survival curves for heart failure occurrence in patients with low (group A) versus high (group B) lactate concentration. HF: heart failure. LC: lactate concentration.

HF-free episode survival rate $(0.95$ versus $0.82, p<0.01$, Figure 2) with no differences in the re-MI episode survival rate $(0.89$ versus $0.88, p=$ ns, Figure 3$)$.

We performed a univariate Cox regression analysis; we then constructed multivariate Cox regression analysis models using the backward stepwise method to predict the occurrence of each endpoint (I, death; II, HF; III, re-MI). A statistically significant influence of LC on death [Hazard Ratio (HR): 1.41, 95\% Confidence Interval (CI) (1.13-1.76), and $p=0.002$ ] and HF occurrence [HR: 1.21, 95\% CI (1.05$1.4)$, and $p=0.007$ ] was reported. The relationship between LC and re-MI during follow-up was not observed. The Cox regression analysis models are presented in Tables 4-6.

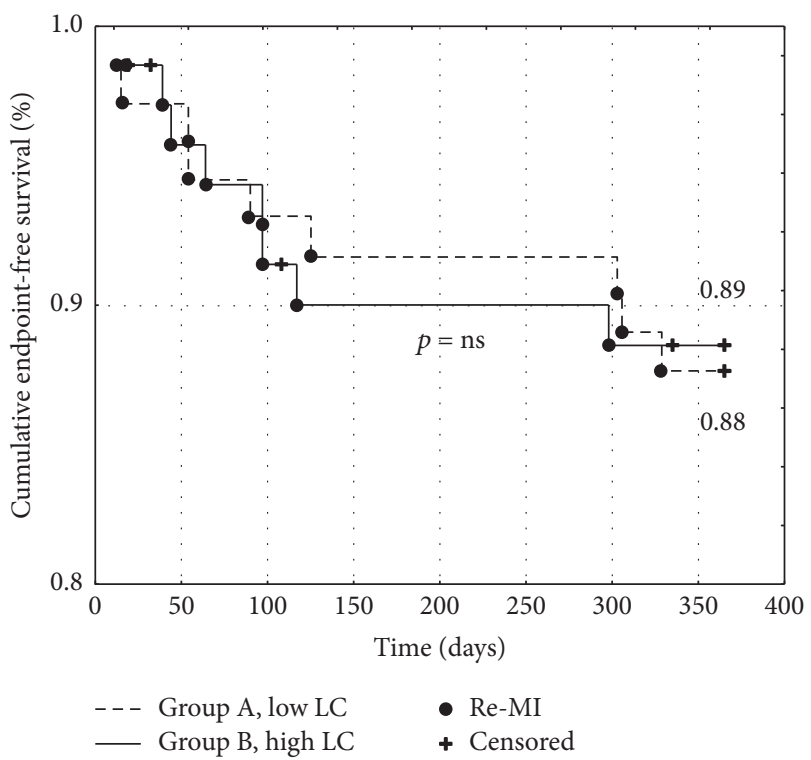

Figure 3: Kaplan-Meier event-free survival curves for recurrent myocardial infarction occurrence in patients with low (group A) versus high (group B) lactate concentration. LC: lactate concentration. Re-MI: recurrent myocardial infarction.

\section{Discussion}

The blood acquisition site substantially impacts LC. What should be considered a significant elevation of LC is in this condition questioned. In our study, a significant elevation was described by the production of more than $2.5 \mathrm{mmol} / \mathrm{l}$; however, LC elevation and its role in the diagnosis of certain clinical states (in addition to prognosis and survival) have not been thoroughly elucidated. Boldt et al. [10] reported the inability to discriminate serious significant differences between LC as assessed using the handheld Accusport diagnostic tool by Roche Diagnostics in addition to the 
TABLE 5: Cox regression model for heart failure occurrence in one year of follow-up.

\begin{tabular}{|c|c|c|c|c|c|c|}
\hline \multirow{2}{*}{ Endpoint II } & \multicolumn{3}{|c|}{ Univariate Cox regression analysis } & \multicolumn{3}{|c|}{ Multivariate Cox regression analysis } \\
\hline & HR & $95 \% \mathrm{CI}$ & $p$ & HR & $95 \% \mathrm{CI}$ & $p$ \\
\hline $\mathrm{AF}$ & 3.53 & $(0.96-12.98)$ & 0.05 & - & - & - \\
\hline BMI & 1.12 & $(1.01-1.24)$ & 0.03 & 1.20 & $(1.07-1.35)$ & 0.002 \\
\hline CREAT & 1.03 & $(1.01-1.04)$ & 0.001 & 1.04 & $(1.02-1.06)$ & 0.0004 \\
\hline GPIIb/IIIa & 3.01 & $(1.03-8.78)$ & 0.04 & 5.00 & $(1.52-16.42)$ & 0.007 \\
\hline IABP & 8.23 & $(1.03-66.06)$ & 0.04 & - & - & - \\
\hline $\mathrm{LC}$ & 1.17 & $(1.04-1.31)$ & 0.01 & 1.21 & $(1.05-1.40)$ & 0.007 \\
\hline $\mathrm{LM}>50 \%$ & 4.53 & $(1.39-14.82)$ & 0.01 & - & - & - \\
\hline Diabetes & 2.73 & $(0.94-7.96)$ & 0.06 & - & - & - \\
\hline Shock & 5.39 & $(1.46-19.88)$ & 0.01 & - & - & - \\
\hline STEMI & 2.59 & $(0.85-7.91)$ & 0.09 & - & - & - \\
\hline WBC & 1.12 & $(0.99-1.25)$ & 0.06 & - & - & - \\
\hline
\end{tabular}

AF: atrial fibrillation. BMI: body mass index. CREAT: creatinine. GPIIb/IIIa: glycoprotein IIb/IIIa inhibitors. IABP: intra-aortic balloon pump. LC: lactate concentration. LM: left main coronary artery. STEMI: ST elevation myocardial infarction. WBC: white blood cells.

TABLE 6: Cox regression model for recurrent myocardial infarction in one year of follow-up.

\begin{tabular}{|c|c|c|c|c|c|c|}
\hline \multirow{2}{*}{ Endpoint III } & \multicolumn{3}{|c|}{ Univariate Cox regression analysis } & \multicolumn{3}{|c|}{ Multivariate Cox regression analysis } \\
\hline & HR & $95 \% \mathrm{CI}$ & $p$ & $\mathrm{HR}$ & $95 \% \mathrm{CI}$ & $p$ \\
\hline CREAT & 1.02 & $(1.01-1.04)$ & 0.00 & 1.02 & $(1.01-1.04)$ & 0.001 \\
\hline DES & 2.54 & $(0.88-7.37)$ & 0.08 & - & - & - \\
\hline Glycemia & 1.13 & $(1.03-1.25)$ & 0.01 & - & - & - \\
\hline HDL & 1.01 & $(1.00-1.02)$ & 0.01 & - & - & - \\
\hline $\mathrm{LAD}>50 \%$ & 4.52 & $(1.00-20.37)$ & 0.05 & 7.08 & $(1.11-44.97)$ & 0.034 \\
\hline LVEF & 0.96 & $(0.92-1.00)$ & 0.03 & - & - & - \\
\hline Number of stents & 1.79 & $(1.04-3.08)$ & 0.03 & 1.69 & $(1.02-2.81)$ & 0.037 \\
\hline Previous SCA & 4.27 & $(0.95-19.26)$ & 0.05 & 23.55 & $(3.25-170.86)$ & 0.001 \\
\hline Smoking & 0.20 & $(0.06-0.71)$ & 0.01 & 0.14 & $(0.04-0.56)$ & 0.005 \\
\hline Stent length & 1.02 & $(1.00-1.04)$ & 0.08 & - & - & - \\
\hline
\end{tabular}

CREAT: creatinine. DES: drug-eluting stent. HDL: high-density lipoproteins. LAD: left anterior descending coronary artery. LVEF: left ventricular ejection fraction. SCA: sudden cardiac arrest.

Chiron Diagnostics 865 and Lactate PAP tools by Analyticon; however, the initial incoherence between the arterial and capillary blood LC assessments were potentially due to technical (different volumes submitted to examination and method of acquisition) and clinical reasons including reduced intravascular osmotic pressure and capillary edema caused by intravenous liquid administration. The delay in LC assessments using the reference method varied between 45 and 168 minutes (mean of 85 minutes) and was twice as expensive as the electronic analyzer with a total cost of five hundred United States dollars (USD). A tight correlation between fingertip and whole blood point-of-care (POC) LC assessment in emergency department patients compared with a standard laboratory analyzer with intraclass correlation coefficients of 0.90 and 0.92 , respectively, was reported by Gaieski et al. [11]. Notably, the time of LC assessment using an electronic analyzer compared with a reference method was 65 minutes shorter in this setting. The impact of LC on infection frequency was previously reported by Claridge et al. [12] and his group in patients hospitalized in the intensive care unit (ICU) after trauma or surgical intervention. In the study, the normalization of LC in time corresponded to the infection occurrence rate (a longer period of time was needed to normalize the higher incidence of infection). The mortality rate was higher in patients with the infection (7.9 versus $1.9 \%, p<0.05$ ) and was indirect proof of LC utility in the early risk assessment and determination of prognosis. Notably, the group of patients with an LC above $2.4 \mathrm{mmol} / \mathrm{L}$ was characterized by a higher rate of death, longer hospital stay, and increased cost of hospitalization. These findings are in accordance with our results, although we examined different group of patients with AMI submitted for invasive coronary angiography and followed up for one year. We believe that this novel approach might be useful in early risk stratification, especially considering low cost of the single LC assessment and its accessibility, although further studies in larger groups of patients and different clinical scenarios would improve the knowledge in the field. Meregalli et al. 
[13] reported the role of $\mathrm{LC}$ in the prognosis of patients after surgical treatment followed by admittance to the ICU. They demonstrated its effectiveness in assessing the risk of death and performed serious complications risk stratification with the area under ROC curves only slightly lower than those for the widely used SAPS II (New Simplified Acute Physiology Scale) [ 0.583 versus 0.705 (for death); 0.646 versus 0.678 (for serious complications)]. LC was thus postulated to be more reliable than other indices of hemodynamic state deterioration, such as heart rate, diuresis and the mean arterial pressure, or metabolic acidosis. The elimination of the clinical signs of shock does not exclude the possibility of hypoperfusion as an important risk factor of serious complications. It is worth mentioning that, after excluding patients with the clinical signs of shock, LC was significantly higher in those who reached endpoints I (8.7 versus 3.8, $p=0.008)$ and II (5.0 versus 3.8, $p=0.028)$. This relationship did not occur for endpoint III (3.7 versus 4.0, $p=\mathrm{ns})$. Howell et al. [14] reported that increased LC $(\geq 4.0 \mathrm{mmol} / \mathrm{l})$ had serious implications for the mortality rate even in normotensive patients. The mortality rate in this group of patients was $15 \%$; however, the patients presenting with septic shock or an LC above $4.0 \mathrm{mmol} / \mathrm{l}$ had a mortality rate of $28.3 \%$, which was significantly higher than that in patients who had neither $(2.5 \%, p<0.0001)$. In a model with good discrimination (AUC $=0.87$ ) consisting of age, blood pressure, malignancy, platelet count, and blood urea nitrogen, LC was considered to be associated with mortality. These findings were also consistent with our results despite the fact that we focused on the potential relationship between LC evaluated during AMI and symptoms of late-onset. The construction of a Cox proportional hazard regression models required the implementation of advanced statistical methods. The ROC-derived cut-off values for each of the three analyzed endpoints varied between 3.3 and $5.4 \mathrm{mmol} / \mathrm{l}$. Jansen et al. [15] analyzed over one hundred patients and reported an increased mortality rate in those with an LC above $3.5 \mathrm{mmol} / \mathrm{l}$ (41 versus $12 \%, p<0.001$, area under ROC curve 0.69 ). These findings are consistent with our results; however, the cardiovascular-related etiology of admission was observed in only $30 \%$ of Jansen's patients (compared with $100 \%$ of our patients). Lazzeri et al. [16] reported a significant impact of LC on prognosis only in patients in the worse hemodynamic state that were assessed on the basis of the Killip-Kimball classification and LVEF assessment; their results were also consistent with our findings. It is worth emphasizing that in contrary to the mentioned study our group consisted in nearly $60 \%$ of NSTEMI patients all of whom had invasive coronary angiography. The LC was significantly elevated (4.7 versus $3.7 \mathrm{mmol} / \mathrm{l}, p<0.025)$ in patients with deteriorated systolic function of the left ventricle that we arbitrarily defined as an LVEF below $45 \%$ in the acute echocardiographic assessment prior to admission to cath lab. Vermeulen et al. [17] reported that an acute hemodynamic state in the patient (expressed by symptoms like increased heart rate, decreased blood pressure, and the presence of diabetes and distal embolization of coronary arteries) significantly impacted LC elevation and the 30 -day mortality rate. These findings were also consistent with our results.

\section{Conclusions}

The point-of-care LC assessment of capillary blood using a handheld analyzer is a safe, easy, and cost-effective method to stratify the risk of late-onset heart failure and death after AMI. Its prognostic potential was conserved irrespective of clinical signs of shock.

\section{Limitations of the Study}

This is a pilot study; thus, the number of patients is relatively small. It was also a single-center study. However, the authors collecting the data did not interfere with the management process. Due to the design of the study and the national law regulations, all patients had to give informed and written consent; hence, the patients who were unconscious were excluded from the study. This could have impacted the potential selection bias and may have resulted in the absence of in-hospital deaths.

\section{Competing Interests}

The authors report no financial relationships or conflict of interests regarding the content in the paper.

\section{Acknowledgments}

The authors thanks are due to the Head Nurse of the Coronary Care Unit, Mrs. Teresa Grzegorczyn, and the nursing team for performing the measurements and to Mr. Sebastian Mielczarek from the Redmed Company for providing the analyzer.

\section{References}

[1] Central Statistical Office of Poland, Statistical Yearbook of Poland, 2012.

[2] R. W. Yeh, S. Sidney, M. Chandra, M. Sorel, J. V. Selby, and A. S. Go, "Population trends in the incidence and outcomes of acute myocardial infarction," The New England Journal of Medicine, vol. 362 , no. 23 , pp. 2155-2165, 2010.

[3] C. J. Terkelsen, J. F. Lassen, B. L. Nørgaard et al., "Mortality rates in patients with ST-elevation vs. non-ST-elevation acute myocardial infarction: observations from an unselected cohort," European Heart Journal, vol. 26, no. 1, pp. 18-26, 2005.

[4] K. Thygesen, J. S. Alpert, A. S. Jaffe et al., "Third universal definition of myocardial infarction," Circulation, vol. 126, no. 16, pp. 2020-2035, 2012.

[5] L. Mandelzweig, A. Battler, V. Boyko et al., "The second euro heart survey on acute coronary syndromes: characteristics, treatment, and outcome of patients with ACS in Europe and the Mediterranean Basin in 2004," European Heart Journal, vol. 27, no. 19, pp. 2285-2293, 2006.

[6] K. A. A. Fox, K. A. Eagle, J. M. Gore, P. G. Steg, and F. A. Anderson, "The global registry of acute coronary events, 1999 to 2009-GRACE," Heart, vol. 96, no. 14, pp. 1095-1101, 2010.

[7] K. W. Scheele, Opuscula Chemica et Physica, 1788.

[8] E. J. O. Kompanje, T. C. Jansen, B. Van Der Hoven, and J. Bakker, "The first demonstration of lactic acid in human blood in shock 
by Johann Joseph Scherer (1814-1869) in January 1843," Intensive Care Medicine, vol. 33, no. 11, pp. 1967-1971, 2007.

[9] M. H. Weil and W. Tang, "Clinical correlates of arterial lactate levels in STEMI patients," Critical Care, vol. 15, no. 1, p. 113, 2011.

[10] J. Boldt, B. Kumle, S. Suttner, and G. Haisch, "Point-of-care (POC) testing of lactate in the intensive care patient. Accuracy, reliability, and costs of different measurement systems," Acta Anaesthesiologica Scandinavica, vol. 45, no. 2, pp. 194-199, 2001.

[11] D. F. Gaieski, B. C. Drumheller, M. Goyal, B. D. Fuchs, F. S. Shofer, and K. Zogby, "Accuracy of handheld point-of-care fingertip lactate measurement in the emergency department," Western Journal of Emergency Medicine, vol. 14, no. 1, pp. 58-62, 2013.

[12] J. A. Claridge, T. D. Crabtree, S. J. Pelletier, K. Butler, R. G. Sawyer, and J. S. Young, "Persistent occult hypoperfusion is associated with a significant increase in infection rate and mortality in major trauma patients," The Journal of Trauma, vol. 48, no. 1, pp. 8-15, 2000.

[13] A. Meregalli, R. P. Oliveira, and G. Friedman, "Occult hypoperfusion is associated with increased mortality in hemodynamically stable, high-risk, surgical patients," Critical Care, vol. 8, no. 2, pp. R60-65, 2004.

[14] M. D. Howell, M. Donnino, P. Clardy, D. Talmor, and N. I. Shapiro, "Occult hypoperfusion and mortality in patients with suspected infection," Intensive Care Medicine, vol. 33, no. 11, pp. 1892-1899, 2007.

[15] T. C. Jansen, J. van Bommel, P. G. Mulder, J. H. Rommes, S. J. M. Schieveld, and J. Bakker, "The prognostic value of blood lactate levels relative to that of vital signs in the pre-hospital setting: a pilot study," Critical Care, vol. 12, no. 6, article R160, 2008.

[16] C. Lazzeri, S. Valente, M. Chiostri, C. Picariello, and G. F. Gensini, "Lactate in the acute phase of ST-elevation myocardial infarction treated with mechanical revascularization: a single-center experience," The American Journal of Emergency Medicine, vol. 30, no. 1, pp. 92-96, 2012.

[17] R. P. Vermeulen, M. Hoekstra, M. W. N. Nijsten et al., "Clinical correlates of arterial lactate levels in patients with ST-segment elevation myocardial infarction at admission: A Descriptive Study," Critical Care, vol. 14, no. 5, article 164, 2010. 


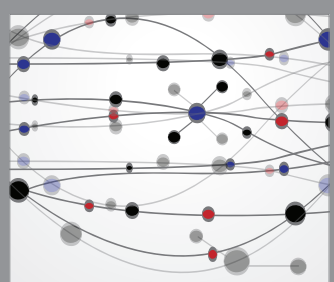

The Scientific World Journal
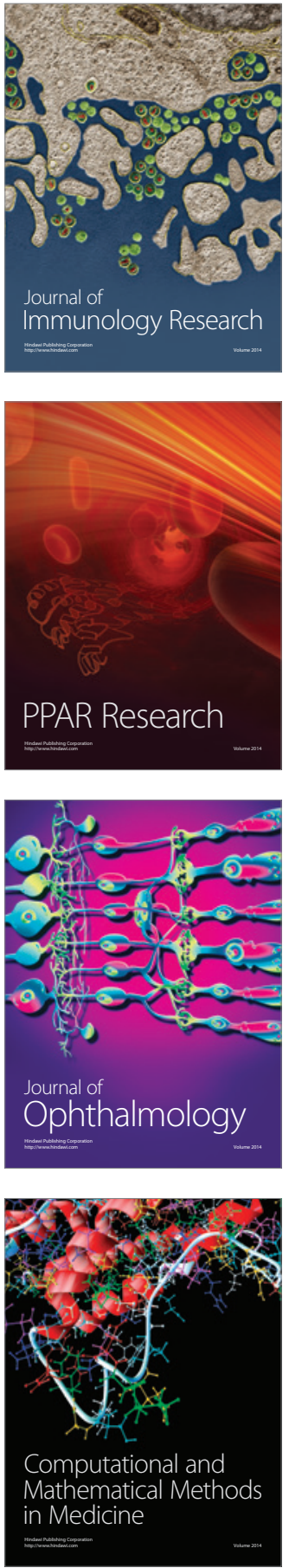

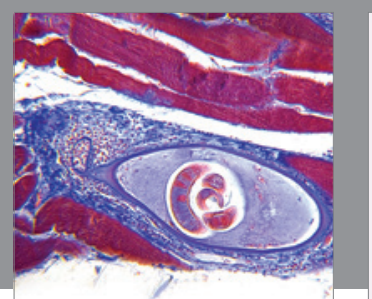

Gastroenterology Research and Practice

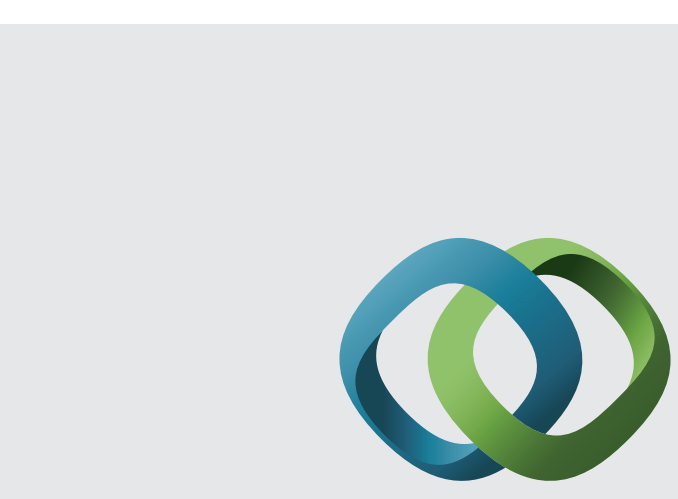

\section{Hindawi}

Submit your manuscripts at

http://www.hindawi.com
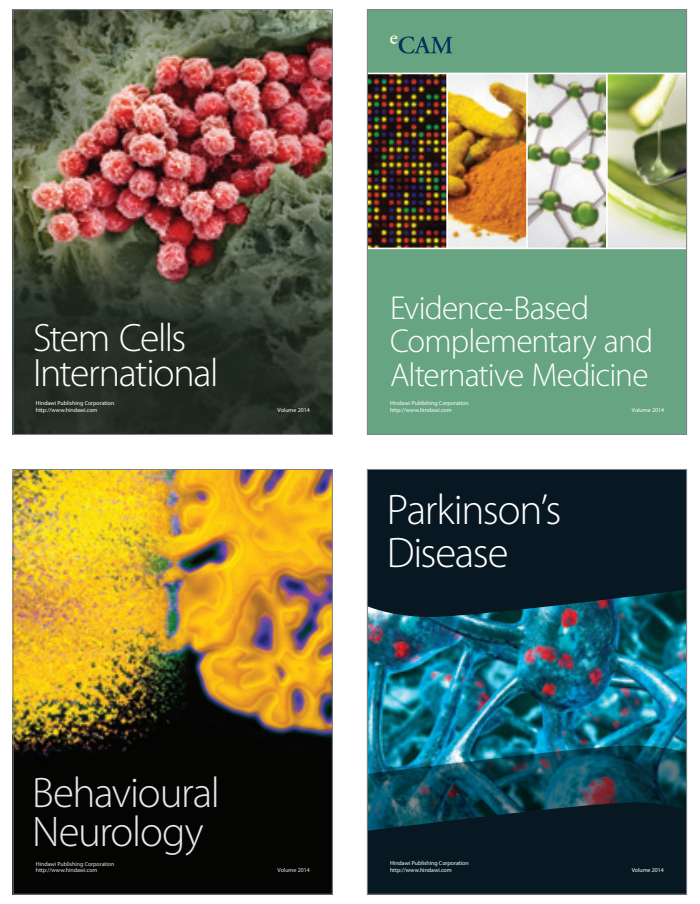
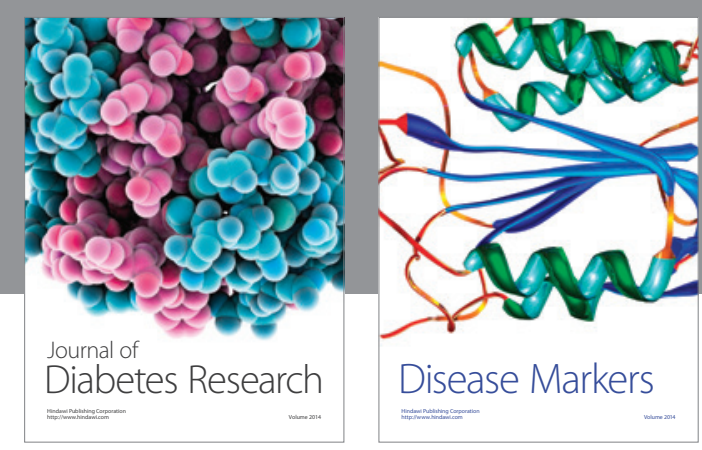

Disease Markers
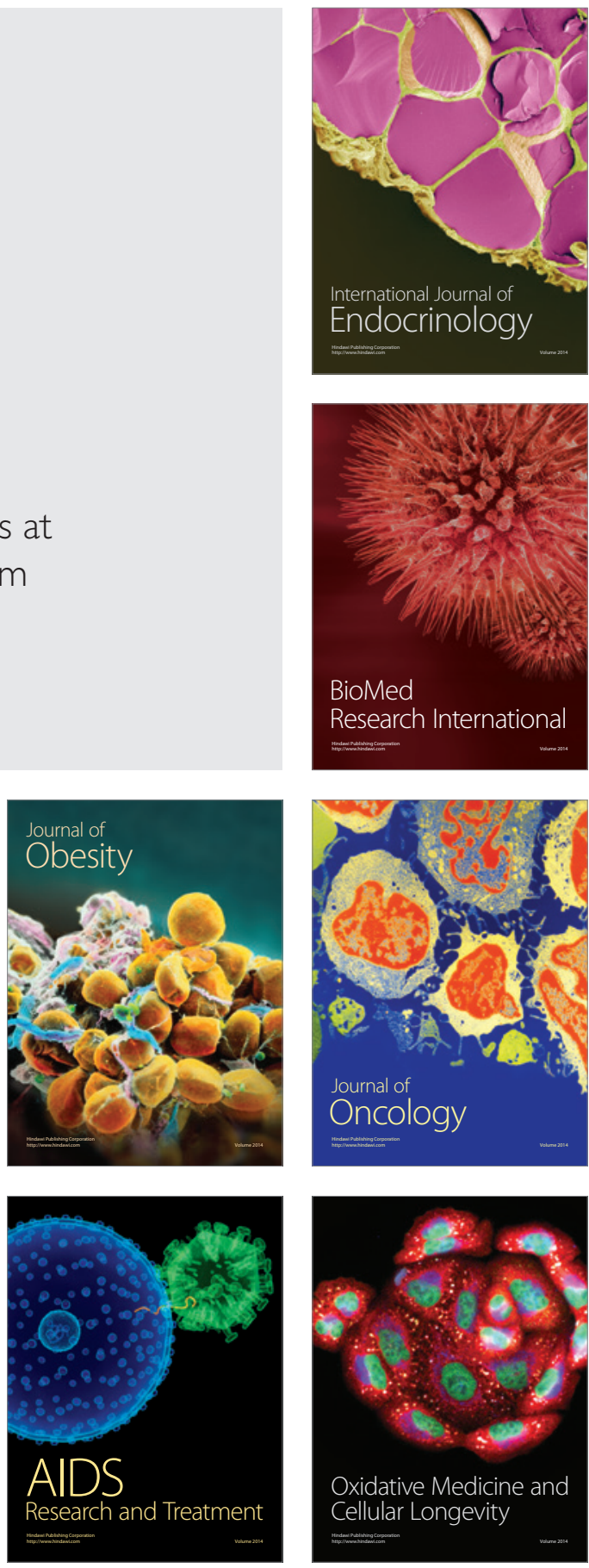